\title{
UCRL-CONF-217015
}

LAWRENCE LIVERMORE N A T IO N A L LABORATORY

\section{Design and Testing of a High Pressure Gas Target for Fast Neutron Resonance Radiography}

W.L Raas, B. Blackburn, E. Boyd, J.M.Hall, G. Kohse, R. Lanza, B. Rusnak, J. Watterson

November 11, 2005

IEEE Nuclear Science Syposium and Medical Imaging Conference

Farado, PR, United States

October 23, 2005 through October 29, 2005 
This document was prepared as an account of work sponsored by an agency of the United States Government. Neither the United States Government nor the University of California nor any of their employees, makes any warranty, express or implied, or assumes any legal liability or responsibility for the accuracy, completeness, or usefulness of any information, apparatus, product, or process disclosed, or represents that its use would not infringe privately owned rights. Reference herein to any specific commercial product, process, or service by trade name, trademark, manufacturer, or otherwise, does not necessarily constitute or imply its endorsement, recommendation, or favoring by the United States Government or the University of California. The views and opinions of authors expressed herein do not necessarily state or reflect those of the United States Government or the University of California, and shall not be used for advertising or product endorsement purposes.

This work was performed under the auspices of the U. S. Department of Energy, by the University of California, Lawrence Livermore National Laboratory under Contract W-7405-Eng.-48. 


\title{
Design and Testing of a High Pressure Gas Target for Fast Neutron Resonance Radiography
}

\author{
Whitney L. Raas, Member, IEEE, Brandon W. Blackburn, Member, IEEE, Erin Boyd, James Hall, Gordon Kohse, \\ Member, IEEE, Richard C. Lanza, Member, IEEE, Brian Rusnak, and John I. W. Watterson
}

\begin{abstract}
A high pressure deuterium gas target has been designed to provide high-flux fast neutrons using the $D(d, n)^{3} \mathrm{He}$ reaction for use as a neutron source. The deuterium gas cell holds $4 \mathrm{~atm} \mathrm{D}_{2}$ gas at $298 \mathrm{~K}$ and is projected to tolerate a beam current of $\sim 50 \mu \mathrm{A}$ of $3.0 \mathrm{MeV}$ deuterons for 8 hours of continuous use. The high-pressure gas cell is designed to provide a fast neutron flux on the order of $10^{5} \mathrm{n} / \mathrm{cm}^{2}$-s at one meter. Measurements of gamma ray production from deuterium impingement have shown tungsten to generate the fewest gamma rays; the primary components of the gas target have been constructed out of tungsten to decrease the number of gamma rays. To accommodate the high gas pressure, thin foil tungsten windows have been structurally reinforced with a tungsten support allowing for more than $60 \%$ beam transmission while greatly increasing the structural reliability of the thin windows. Extensive simulation and experimental testing have demonstrated the heating tolerances of the gas target thin windows and have shown that the peak temperature of the thin foils does not exceed $600{ }^{\circ} \mathrm{C}$, while the edges of the foil do not exceed $100{ }^{\circ} \mathrm{C}$, well within the limits of the foil windows and the gas sealing structures.
\end{abstract}

\section{INTRODUCTION}

$\mathrm{N}$ EUTRON Resonance Radiography (NRR) is under investigation as a non-invasive, non-destructive means to determine elemental composition of concealed objects using fast neutrons. Fast neutrons for NRR are produced using the $\mathrm{D}(\mathrm{d}, \mathrm{n})^{3} \mathrm{He}$ reaction; the incoming deuterons are accelerated to 2.5 MeV using a small radio frequency quadrupole (RFQ) accelerator. Previous studies have concluded that a windowed deuterium gas target is preferable to a solid target imbedded with deuterium, and to windowless, differentially pumped gas targets [1] in this application. NRR requires neutrons with specific characteristics in order to perform properly; namely, the neutron beam must be mono-energetic over a $10^{\circ}$ angular span, and the neutron flux must be in excess of $10^{4} \mathrm{n} / \mathrm{cm}^{2} / \mathrm{s}$ at a distance of one meter from the source. Neutron detection is accomplished using solid plastic scintillator, coupled to a CCD camera for direct imaging or to a light guide and

Manuscript received October 11, 2005. This work was supported by the US Government, Technical Support Working Group and by the Transportation Security Administration.

W. Raas, B. Blackburn, E. Boyd, G. Kohse, and R. Lanza, are with the Department of Nuclear Science and Engineering at the Massachusetts Institute of Technology, Cambridge, MA 02139 USA (telephone: 617-253-5730, email: wraas@mit.edu).

J. Hall and B. Rusnak are with Lawrence Livermore National Laboratory, 7000 East Ave., MS L050 Livermore, CA, 94550.

$\mathrm{J}$. Watterson is with the Department of Nuclear Science and Engineering at the Massachusetts Institute of Technology, Cambridge, MA 02139 USA, on leave from the University of Witwatersrand, Johannesburg, South Africa. photomultiplier tube for neutron/gamma discrimination and neutron counting [2].

The use of NRR in a deployable explosives detection system is based on its ability to determine the elemental composition of objects reliably in equal or less time than current detection systems. In order to accomplish this, the neutron flux from the target must be high to reduce the imaging time necessary to acquire clear images. The gas target, as the neutron source, plays the most important role in the viability of NRR. Significant work has been put into development of a new gas target that will support a high neutron flux.

In addition to its role as neutron source, the gas target is also a significant source of gamma rays, which may obscure the contrast in the final neutron images. A reduction in the gamma flux is essential, especially when a CCD camera is used for imaging, to improve elemental discrimination in the presence of a mixed radiation field. Although no gamma rays are produced in the $\mathrm{D}(\mathrm{d}, \mathrm{n})^{3} \mathrm{He}$ reaction, the gas target is still a considerable source of gamma rays as a large amount of the energy of the incident deuteron beam is lost to metallic components of the target.

This paper discusses the improved gas target design and its implementation. Section II describes the gas target design in detail. Sections III and IV are a summary of the experimental analysis of materials for use in the gas target, including the gamma ray production from various metals and the energy reduction in the deuteron beam caused by the foil windows and the deuterium gas cell itself. Sections V and VI provide details of the structural and mechanical properties of the gas target when operated under realistic conditions.

\section{Gas TARget Design}

The gas target was designed to tolerate high pressure and high currents to produce a significant neutron flux. There are three separate important features of the gas target: the deuterium gas chamber, the beam stop, and the beam scraper assembly. The deuterium gas containment space is a tungstenlined chamber with thin tungsten windows front and back supported by "strongbacks" $(0.25 \mathrm{~mm}$ thick tungsten with circular holes in a pattern to produce about $60-80 \%$ open area for beam transmission). The window thickness is 5-7 $\mu \mathrm{m}$ and the target will contain 3-7 atm-cm of deuterium. Aluminum cooling fins surround the target and are cooled by circulating argon to facilitate forced heat convection from the gas cell. The beam stop behind the rear tungsten window assembly contains circulating argon gas in an aluminum housing. The argon helps cool the target chamber and produces fewer gamma rays than a metal beamstop. An entrance beam scraper 
composed of four segments of tungsten is provided to shape and center the beam. The target module can be replaced independently of the beam scraper module and target thin windows are replaceable. The target design is shown in Fig. 1 below. A detailed image of the gas cell and the strongback are shown in Fig. $2 a$ and $2 b$.

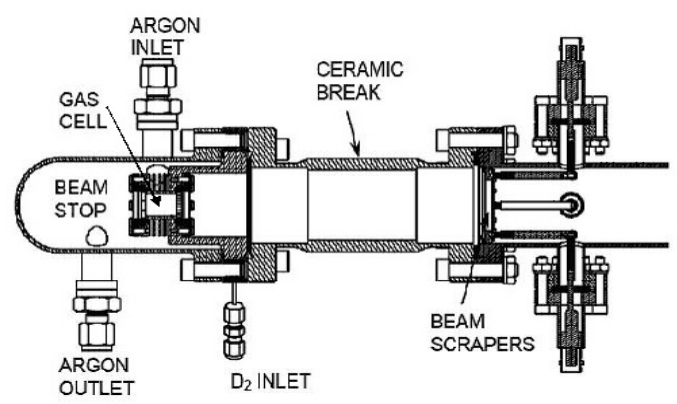

Fig. 1. Gas target layout.

(a)

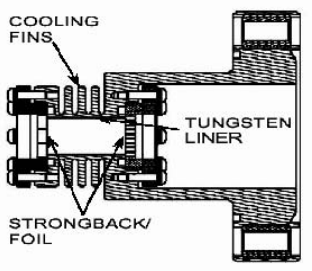

(b)

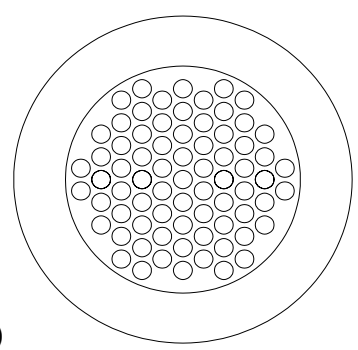

Fig. 2. Detailed image of (a) the gas cell, and (b) the strongback support structure for the thin gas cell windows.

The improvements to the gas cell and the beam stop are the most important feature of the gas target. The thin tungsten windows of the deuterium containment cell provide isolation from the beamline vacuum and the beam stop without significantly affecting the energy of the incident deuteron beam, discussed further in Section IV. The strongback provides the support structure necessary for the high pressure of the gas cell and the high deuteron beam current that supply an increased neutron flux. For this application of NRR, a very small beam spot is not required; a beam spot on the order of $1 \mathrm{~cm}$ in diameter is quite adequate. This large diameter beam spot distributes heat and energy over a larger area of the foil window, increasing the ability of the window to withstand high currents. The aluminum cooling fins increase heat conduction from the foil windows and strongbacks to the circulating argon, which allows for further increases in deuteron current. The argon beam stop is also a significant improvement over previous gas target designs. The argon is designed to be cooled and circulated, providing a means of heat removal as well as functioning in its primary role as deuteron beam stop. The argon is held at the same pressure as the gas cell to reduce stress on the back foil window, and flowed at a rate of 5-10 cfm through the beam stop.

\section{MATERIALS CHARACTERIZATION}

Materials for use in a deuterium gas target must meet the following requirements. They must have high melting points to withstand the high temperatures caused by heat deposition from the accelerated deuterium ions, they must have high stress tolerance to avoid premature failure, they must be fabricable into the necessary components, and, finally, they must produce the minimum number of gamma rays. The neutron detection system is extremely sensitive to gamma rays, which negatively affect image quality and the ability to determine the elemental constituents of objects under investigation. Scintillators that are responsive to fast neutrons generally have an order of magnitude or greater response to gamma rays, even at low energies [3]. Consequently, gamma rays that interact in the scintillator will be the overwhelming signal that reaches the CCD camera, rather than the desired scintillation photons from neutrons. Minimization of gamma ray production is a first step towards improving the quality of the final images created by scintillation photons detected by the CCD.

Besides the beamline material itself, components of the gas target that are exposed to deuterons include the thin foil windows, the metal strongbacks that support the foil windows, and the beamstop which ultimately absorbs any deuterons which do not produce neutrons or are not absorbed in the strongbacks. The metals chosen for evaluation were nickel, iron, stainless steel, tungsten, machinable (heavy metal) tungsten, molybdenum, and gold. A summary of relevant properties is shown in Table I.

TABLE I

MATERIAL PROPERTIES

\begin{tabular}{llll}
\hline Material & $\begin{array}{l}\text { Purity }(\%) / \\
\text { Composition }\end{array}$ & $\begin{array}{l}\text { Density } \\
\left(\mathrm{g} / \mathrm{cm}^{3}\right)\end{array}$ & $\begin{array}{l}\text { Melting } \\
\text { Point }\left({ }^{\circ} \mathrm{C}\right)\end{array}$ \\
\hline $\begin{array}{l}\text { Stainless } \\
\text { Steel }\end{array}$ & AISI 316 & 8.17 & 1399 \\
\hline Gold & 99.95 & 19.32 & 1064.2 \\
\hline Nickel & 99.95 & 8.908 & 1455 \\
\hline Tungsten & 99.998 & 19.30 & 3410 \\
\hline $\begin{array}{l}\text { Machinable } \\
\text { Tungsten }\end{array}$ & $90 \% \mathrm{~W}$, & 17.42 & 1200 \\
\hline Molybdenum & 99.95 & 10.22 & 2623 \\
\hline Iron & 99.95 & 7.87 & 1538 \\
\hline
\end{tabular}

These metals were chosen for a variety of reasons. Stainless steel is the primary component of the beamline components; some deuterium impingement is likely despite the best efforts in focusing the beam. Nickel and iron were chosen as separate materials to determine the contributions to the stainless steel spectrum. Tungsten is a primary candidate for thin foil windows for the gas cell due to its high melting point and ability to withstand mechanical stress; however, it is an extremely difficult material to machine and quite expensive for high purity material. Machinable tungsten is a composite material made from 90\% tungsten, $7 \%$ nickel, and 3\% iron; the combination results in a material that is much more amenable to fabrication but retains most of the strength and heat resistance of pure tungsten. Molybdenum has similar 
properties to tungsten, while gold has been used as the beam stop in many gas targets.

Deuteron-induced gamma production was determined using the Massachusetts Institute of Technology's Laboratory for Accelerator Beam Applications [4] tandem accelerator to provide a beam of $2.5 \mathrm{MeV}$ deuterons. Thin foils, thick enough to fully stop the entire deuteron beam, of each material were placed directly in line with the deuteron beam and the gamma ray spectrum was measured using a high-purity germanium detector. The detector was shielded with lead to create a collimated opening pointed directly at the metal foil at a $90^{\circ}$ angle to the beam direction to ensure that the gamma rays produced by the metal in question were detected. The results of the gamma production experiments are shown in Fig. 3. The spectra have been normalized to irradiation time and beam current for comparison.

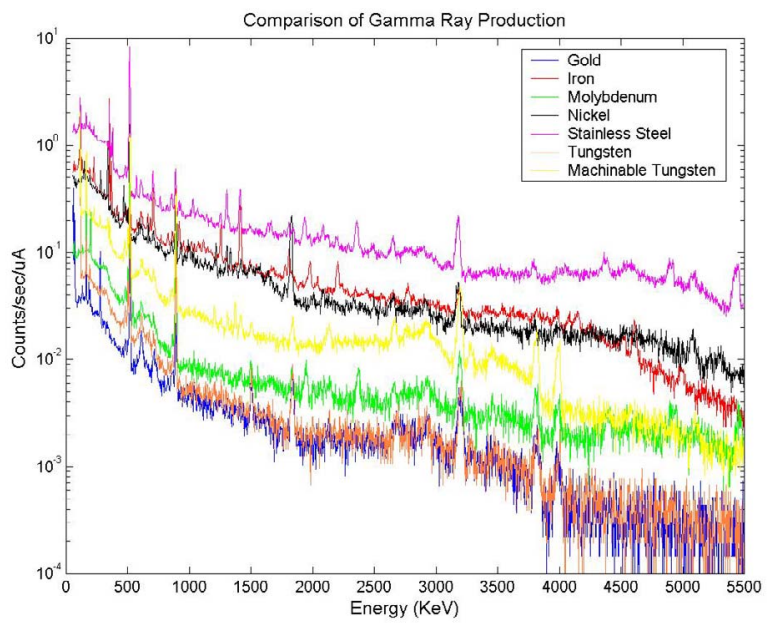

Fig. 3. Gamma ray production from $2.5 \mathrm{MeV}$ deuteron impingement on metal.

Fig. 3 clearly shows the significant contribution to the gamma flux from stainless steel. However, the relatively low gamma production both in terms of overall flux and highenergy gamma rays from pure tungsten and molybdenum, coupled with their favorable structural properties outlined in Table 1, make these the ideal materials for use in the beamfacing components of the gas target. Because of its high melting point, high tolerance to mechanical stresses, and low gamma ray production, pure tungsten was chosen for the thin foil windows and window supports of the gas cell, the beam scraper material, and as a lining of the gas cell to ensure that the incident deuterons only come into contact with tungsten. Pinhole-free molybdenum foils are more readily available than tungsten foils and can be substituted for tungsten without undue loss of performance. The somewhat lower melting point and mechanical strength of molybdenum compared to tungsten requires the use of slightly thicker windows, but this is compensated for by the linear energy loss for deuterons.

\section{Deuteron Energy Loss In The Gas CelL}

As the incident deuteron beam passes through the gas cell foil window, energy loss due to electromagnetic interactions between the deuterium ion and the metal foil cause the deuteron to interact with the deuterium gas at a lower initial energy. The energy of deuteron beam is also spread by interactions within the gas cell. Broadening of the energy spectrum of the incident deuteron beam causes broadening in the energy of the neutrons produced. For applications such as $\mathrm{NRR}$, in which a monoenergetic neutron beam is required, the energy spread of the deuterons throughout the gas target system is a vital quantity.

The deuteron energy loss can be calculated using the freely available SRIM/TRIM computational software [5]. Simulations of the energy loss of a $2.5 \mathrm{MeV}$ deuteron beam in a $5 \mu \mathrm{m}$ tungsten window and a $7 \mu \mathrm{m}$ molybdenum thin window show average deuteron energy after the foils of $1.89 \pm$ $0.040 \mathrm{MeV}$ and $1.83 \pm 0.030 \mathrm{MeV}$, respectively, as shown in Fig. 4. Due to the lower density of molybdenum, the broadening of the energy spectrum is less significant than that of the tungsten window, although the thicker foil increases the average energy loss.

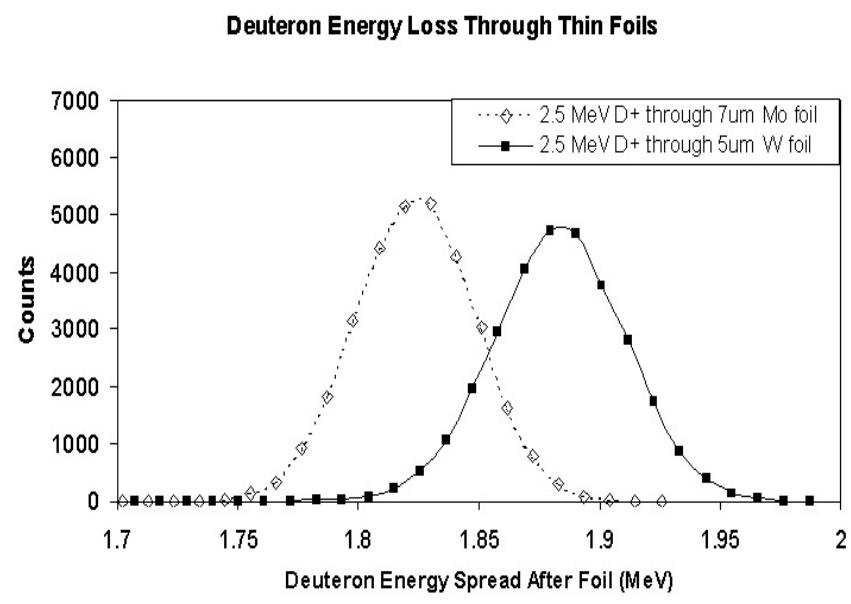

Fig. 4. Energy broadening of the $2.5 \mathrm{MeV}$ deuteron beam through gas cell thin foil windows.

The deuteron energy spread is further broadened through the deuterium gas in the cell, which contributes most significantly to the neutron energy spread. Deuterium ions can react with the deuterium gas to produce neutrons throughout the length of the cell, creating an energy spread in the neutron beam between those neutrons created at the front of the target and those created at the back. Using SRIM, the energy spread of initial deuterons of $2.5 \mathrm{MeV}$ (including the energy drop from the foil windows shown in Fig. 4) is $420 \mathrm{keV}$ for the 5 $\mu \mathrm{m}$ tungsten window and $440 \mathrm{keV}$ for the $7 \mu \mathrm{m}$ molybdenum window. For the purposes of NRR, this spread in energy of the neutron beam is acceptable, so long as the object under investigation spans an angle of less than $10^{\circ}$.

\section{HeAT AND TEMPERATURE CHARACTERISTICS}

As the deuteron beam passes through the thin foil windows on either side of the gas cell, the deuterons lose energy and deposit heat into the foil windows. Heat transfer from the foils is difficult due to their thinness and the lack of a convection transfer mechanism on the beamline side of the target. The lack of efficient heat removal results in a rapid rise in 
temperature of localized areas of the foil window, contributing to mechanical stress and increasing the likelihood of foil failure. Most of the energy removed from the deuteron beam is deposited in the front tungsten foil; this fact and the higher mechanical stress on the front foil and strongback led us to focus primarily on heating of the front foil.

The finite element code ADINA $^{\text {TM }}$ was used to simulate the thermal heat load of the foil windows. A $5 \mu \mathrm{m}$ foil having a radius equal to that of the strongback was modeled using $3 \mathrm{D}$ elements. Initial models did not consider conduction into the strongback, but they did approximate the contact resistance with the aluminum housing by using an equivalent resistance of $2.0 \times 10^{-4} \mathrm{~m}^{2}-\mathrm{K} / \mathrm{W}$. This is a conservative assumption since conduction into the strongback would be an additional path for heat removal from the front of the foil. Fully temperature dependent thermal properties for pure tungsten were used. Heating was modeled using a uniform heat flux onto the foil. In order to simulate the shadowing effect of the strongback, heat loads were applied to areas of the foil not blocked from the beam by the strongback. Temperature dependent natural convection heat transfer coefficients of $10-100 \mathrm{~W} / \mathrm{m}^{2}-\mathrm{K}$ were placed on the area of the foil which would be exposed to the deuterium gas in the target cell. These coefficients were derived from standard Rayleigh number correlations used to model natural convection from a flat plate. As expected, the natural convection from the foil is small due to the small dimensions of the deuterium cell. The temperature of the gas in the cell was held at $50{ }^{\circ} \mathrm{C}$. The front side of the foil not shielded by the strongback was allowed to radiate into a vacuum held at $25^{\circ} \mathrm{C}$.

A beam consisting of $2.5 \mathrm{MeV}$ deuterons was used in the simulations. Beam currents from 5 to $40 \mu \mathrm{A}$ were simulated in $5 \mu \mathrm{A}$ increments. The resulting temperature vs. current profile is shown in Fig. 7. At a beam current of $40 \mu \mathrm{A}$, the peak foil temperature reached a limit of $560{ }^{\circ} \mathrm{C}$, while the edges of the foil did not exceed $80{ }^{\circ} \mathrm{C}$. Simulations which increased the heat transfer coefficient, simulating a constant deuterium flow for forced convection, resulted in a significant drop in foil temperature on the order of $200{ }^{\circ} \mathrm{C}$ at $20 \mu \mathrm{A}$. Providing deuterium flow through the gas cell will significantly increase the beam current the foil windows can withstand without failure.

The ADINA ${ }^{\mathrm{TM}}$ simulations allowed us to gain some understanding of the expected behavior of the gas target, but realistic experiments to verify the simulations were also undertaken. A series of measurements were made using an infrared camera to create an image of the temperature distribution on the target window and strongback and verify the results of the simulations. The temperatures observed were used to correlate experimental results with simulations of the gas target. The camera was able to measure temperatures up to $580{ }^{\circ} \mathrm{C}$ with $0.3 \mathrm{~mm}$ spatial resolution. A front surface mirror was used for imaging to avoid placing the camera directly in the deuteron beam. Estimates based on emissivity experiments for heated tungsten foils were used to assign an emissivity value of 0.3 . All of the measurements described were made with $5 \mu \mathrm{m}$ tungsten windows and strongbacks with the small hole pattern (Fig. 2b).
A $5 \mu \mathrm{A}$ beam of $2.5 \mathrm{MeV}$ deuterons was run through the gas cell to measure the resulting temperature rise on the rear strongback and foil at twenty second intervals with no cooling mechanism applied. The foil is uniformly heated through its thickness while the strongback is directly heated by the beam only on the side opposite to the side imaged to a depth of about $15 \mu \mathrm{m}$. The resolution of the camera allowed us to observe the temperature difference between the thin foil window and the strongback. Fig. 5 shows a representative image from these tests. The strongback is clearly at a lower temperature than the foil.

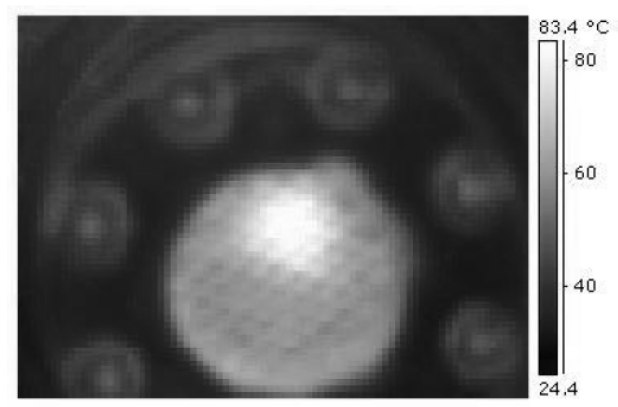

Fig. 5. Infrared image of rear tungsten foil and strongback irradiated by a $5 \mu \mathrm{A}, 2.5 \mathrm{MeV}$ deuteron beam. Peak temperature of the foil is $83{ }^{\circ} \mathrm{C}$. Differences in the heating of the foil vs. the strongback are clearly noticeable.

A more extensive series of measurements was made of the gas cell side of the front window foil with the front strongback placed on the beamline side of the gas cell. In order to simulate the cooling that will be provided by circulating the argon in the beam stop around the gas cell, forced air cooling was applied to the cooling fins of the gas cell. Initially, a $5 \mu \mathrm{A}$ beam of $2.5 \mathrm{MeV}$ deuterons was applied and images of the front foil were recorded every ten seconds. As the foil was heated and stabilized, the current was increased in $5 \mu \mathrm{A}$ increments and the infrared images were recorded, again at 10 second intervals. The temperature of the foil was recorded for beam currents of 5 to $35 \mu \mathrm{A}$ in $5 \mu \mathrm{A}$ intervals. Fig. 6 below is an image of the foil irradiated by a $2.5 \mathrm{MeV}, 35 \mu \mathrm{A}$ deuteron beam. The maximum temperature was recorded at $552{ }^{\circ} \mathrm{C}$ at the center of the beam spot, well below any structural limits of the tungsten foil. The temperature of the edge of the foil was approximately $100^{\circ} \mathrm{C}$.

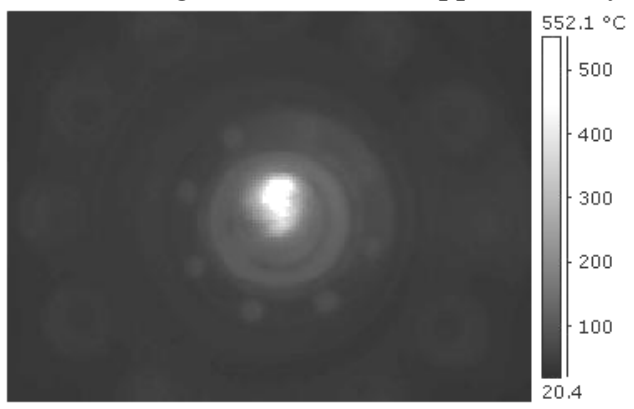

Fig. 6. Infrared image of front foil under a $35 \mu \mathrm{A}, 2.5 \mathrm{MeV}$ deuteron beam.

The temperature rise as a function of beam current was also addressed using the same set of experiments. Two measurements were used to determine the temperature dependence on the beam current: the maximum temperature of 
the foil and an average of the hottest area of the foil. The linear dependence of temperature on the beam current was confirmed through these measurements, as well as establishing the speed with which the foil increased in temperature and rapidly reached equilibrium at the new, higher temperature within fifteen seconds. In addition, the experimental trend of temperature vs. current was compared to simulations of a 2.5 $\mathrm{MeV}$ deuteron beam and was shown to agree favorably with expected values, as shown in Fig. 7.

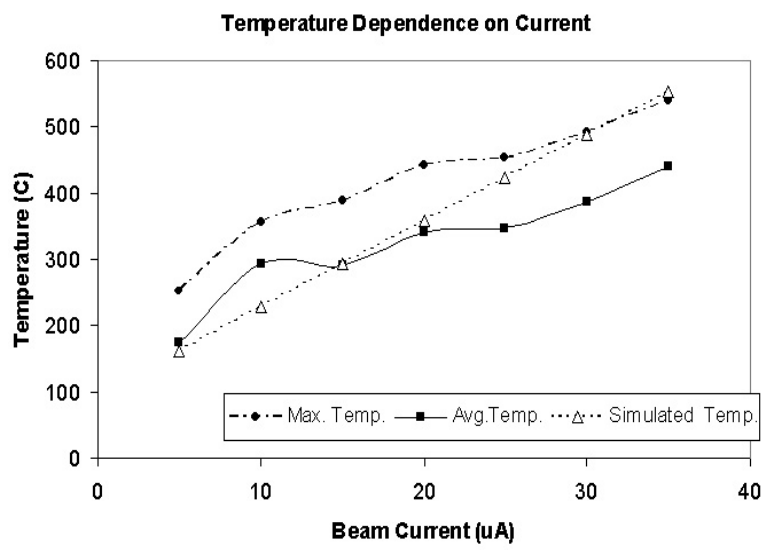

Fig. 7. Measurements of the front foil temperature as a function of beam current compared to simulated values. All deuterons were of energy 2.5 $\mathrm{MeV}$.

The simulations of heating and stress on the gas cell foil windows and the experimental measurements of the actual temperature show that the gas target will perform reliably and as expected with no failure of the windows or the excessive heating of the temperature-sensitive components of the gas target. Conservative estimates indicate that the gas target will withstand an average deuteron beam current of $50 \mu \mathrm{m}$ using 3.0 $\mathrm{MeV}$ deuterons; the experimental results shown here with limited cooling of the gas target indicate that this current should not be difficult to attain.

\section{CONCLUSIONS}

A new windowed gas target design has been designed as an accelerator-based, high-flux fast neutron source for neutron resonance radiography. The target consists of a high-pressure gas cell holding 4 atm of deuterium gas for use in the $\mathrm{D}(\mathrm{d}, \mathrm{n})^{3} \mathrm{He}$ reaction. The gas cell is separated from the accelerator beamline by thin tungsten or molybdenum foil windows which must withstand the intense heating and mechanical stresses on the incident deuteron beam. As an extra support mechanism, the windows are strengthened by thick tungsten strongbacks, consisting of a pattern of small holes allowing for 60 percent transmission of the deuteron beam. As gamma rays are detrimental to the efficacy of NRR, experimental analysis of gamma ray production in the gas target was investigated. It was established that tungsten, gold, and molybdenum produce the fewest gamma rays under deuteron bombardment. Due to its advantageous thermal and mechanical properties, primary components of the gas target have been constructed of tungsten. Extensive simulations and experiments to demonstrate the thermal and mechanical reaction of the thin tungsten foil windows have shown that the gas cell has the ability to withstand $2.5 \mathrm{MeV}$ deuterons at a beam current of at least $35 \mu \mathrm{A}$ for extended periods. A further increase in beam current, and therefore in neutron flux, can be accomplished by circulating the deuterium gas to provide forced convection for heat removal from the foil window on the inner side of the gas cell.

\section{ACKNOWLEDGMENT}

The authors gratefully acknowledge the help of Gongyin Chen and Dave Perticone for their expertise and many useful discussions. We also would like to thank Ashley Finan and Jack Styczynski for their help in the preparation of thin tungsten foils.

The views and opinions presented in this document are those of the author(s) and do not necessarily represent the official position or policies of the US Government.

\section{REFERENCES}

[1] G. Chen, "Neutron Resonance Radiography for Elemental Imaging: Theory and Applications", Ph.D. Dissertation, Massachusetts Institute of Technology, 2001.

[2] See W. Raas, et al., "Neutron Resonance Radiography for Explosives Detection: Technical Challenges", these Proceedings.

[3] Eljen Technology, Sweetwater, TX: http://www.eljentechnology.com.

[4] B. W. Blackburn, "Characterization of a High-Current Tandem Accelerator and the Associated Development of a Water-Cooled Beryllium Target for the Production of Intense Neutron Beams", M. S. Thesis, Massachusetts Institute of Technology, 1997.

[5] SRIM/TRIM online manual, available at http://www.srim.org. 